PSU-TH/239

\title{
$Q \bar{Q} g$ contribution to diffractive $J / \psi$ electroproduction
}

\author{
F. Hautmann \\ Department of Physics, Pennsylvania State University \\ University Park PA 16802
}

\begin{abstract}
We study the diffractive electroproduction of quarkonia from quark-antiquark-gluon states in the photon wave function. We show that these states contribute to the leading-power and leading-logarithm level. We suggest the measurement of $J / \psi$ production via inelastic diffraction to study color-transparency and color-opacity effects in the diffractive gluon distribution.
\end{abstract}

Keywords: QCD, Deep Inelastic Scattering. 


\section{Contents}

1. Introduction 1

2. $J / \psi$ production by $Q \bar{Q} g$ states: the $s$-channel point of view 2

3. The $t$-channel point of view 5

4. $Q^{2} \neq 0$ case $\quad 7$

5. Discussion $\quad 8$

\section{Introduction}

$J / \psi$ mesons are produced copiously via hard-diffractive reactions in lepton-hadron collisions at high energies. In these processes, the initial hadron emerges intact in the final state and the meson is produced via a short-distance interaction, involving momentum transfers of the order of the heavy quark mass or, for highly off-shell photons, the photon virtuality.

The elastic process $\gamma+A \rightarrow V+A^{\prime}$, where $A$ and $A^{\prime}$ are the initial and final hadrons and $V$ is the quarkonium, has been studied extensively at HERA $[1,2$, $3]$. This process is computable in $\mathrm{QCD}[4,5,6]$ in the sense that a short-distance coefficient, calculable as a power expansion in $\alpha_{s}$, can be factorized [7] from a parton density describing the long-distance dynamics of the hadronic state $A$ and a wave function describing the long-distance dynamics of the produced bound state. A typical leading-order graph is shown in Fig. 1. The parton density probed by this process is a nondiagonal parton density $[7,8,9,10]$ :

$$
\phi_{g}\left(\xi, x_{\mathbb{P}}, t, \mu\right)=\frac{1}{2 \pi \xi p_{A}^{+}} \int d y^{-} e^{i \xi p_{A}^{+} y^{-}}\left\langle A^{\prime}\left|\widetilde{G}_{a}(0)^{+j} \widetilde{G}_{a}\left(0, y^{-}, \mathbf{0}\right)^{+j}\right| A\right\rangle
$$

where $p_{A}^{+}$is the lightcone plus momentum of hadron $A, x_{\mathbb{P}}=1-p_{A^{\prime}}^{+} / p_{A}^{+}, t=$ $\left(p_{A}-p_{A^{\prime}}\right)^{2}$. The operator $\widetilde{G}\left(0, y^{-}, \mathbf{0}\right)$ is the gluon field strength multiplied by a path-ordered exponential of the line integral of the color potential from $y^{-}$to $\infty$. The ultraviolet divergences from the operator product are renormalized at the scale $\mu$.

In this paper we are concerned with the production of quarkonia by inelastic diffraction, $\gamma+A \rightarrow V+X+A^{\prime}$, a process that has not been considered so far but could be measured at HERA and at future lepton-proton and lepton-nucleus 
colliders. This process probes a different parton density than (1.1): see Eq. (2.1) ahead. As we will see, for sufficiently large values of the hard-scattering scale its cross section is enhanced by two powers of the hard scale compared to the case of Fig. 1.

In the rest frame of the target (see Sec. 2), the elastic production is dominated by fluctuations of the incoming photon into quark-antiquark states, while the process we consider in this paper is dominated by fluctuations into quark-antiquark-gluon states. The main interest in measuring this process is to observe effects of the coupling of these states to the target's soft color field. The color dipole interaction is stronger for $Q \bar{Q} g$ states than for $Q \bar{Q}$ states. We discuss consequences of this in Sec. 5 .

The contents of the paper is as follows. We present the physical picture of the process in Sec. 2. This discussion uses the target rest frame and gives an " $s$-channel view" of the process, based on the evolution of the system into which the incoming photon dissociates. Then we relate the evolution of this system to diffractive matrix elements of gluon field operators, and in Secs. 3 and 4 we give factorization formulas for the quarkonium cross sections. This formulation corresponds to a " $t$-channel view" of the process. We use these results to contrast the scaling behaviors of the elastic and inelastic contributions. Sec. 5 contains a concluding discussion, focusing on the possibility that diffractive interactions of $Q \bar{Q} g$ states with the target be dominated by small transparency lengths, and on the implications of this for the diffractive gluon distribution. The analysis of this paper applies to both $J / \psi$ and $\Upsilon$ mesons. Numerical evaluations of the cross sections are left to future work.

\section{2. $J / \psi$ production by $Q \bar{Q} g$ states: the $s$-channel point of view}

In this section we work in the rest frame of the target and present the physical picture for the production of quarkonia from quark-antiquark-gluon states in the photon wave function.

As is well known, the spacetime structure $[11,12]$ of hard-diffractive processes in electroproduction looks especially simple in the target rest frame. In this frame, at lightcone times far in the past a highly energetic photon dissociates into a partonic system. Much later, this system interacts with the color field of the target, mainly through its color dipole moment [12]. The physics of the parton densities is reexpressed in this frame in terms of the dipole-target scattering (see reviews in [13, 14]). The elastic production of quarkonia (Fig. 1) has been analyzed in this context in Refs. $[4,5,6]$. This process is dominated by the contribution of the quark-antiquark component in the photon wave function.

Fig. 2 depicts a typical inelastic contribution to the diffractive production of quarkonia. In the target rest frame, a quark-antiquark-gluon state is created by the photon well outside of the target hadron. This system has large minus momentum and travels a long distance before interacting with the target's color field. (This 
interaction is schematically represented in Fig. 2 as two-gluon exchange. In general, it includes any number of soft gluons and is nonperturbative. Note also that contributions in which $k_{1}$ and $k_{2}$ attach to the quark lines in the upper subgraph are to be considered together with the contribution depicted in Fig. 2. One needs to include them to construct gauge-invariant quantities.) Over long times, the quark and antiquark bind into a quarkonium. The emission of two gluons into the final state in Fig. 2 is required by color conservation. The interaction of the fast-moving parton system with the target's field leaves hadron $A$ almost intact, and produces a final state consisting of the quarkonium plus jets plus the diffracted hadron.

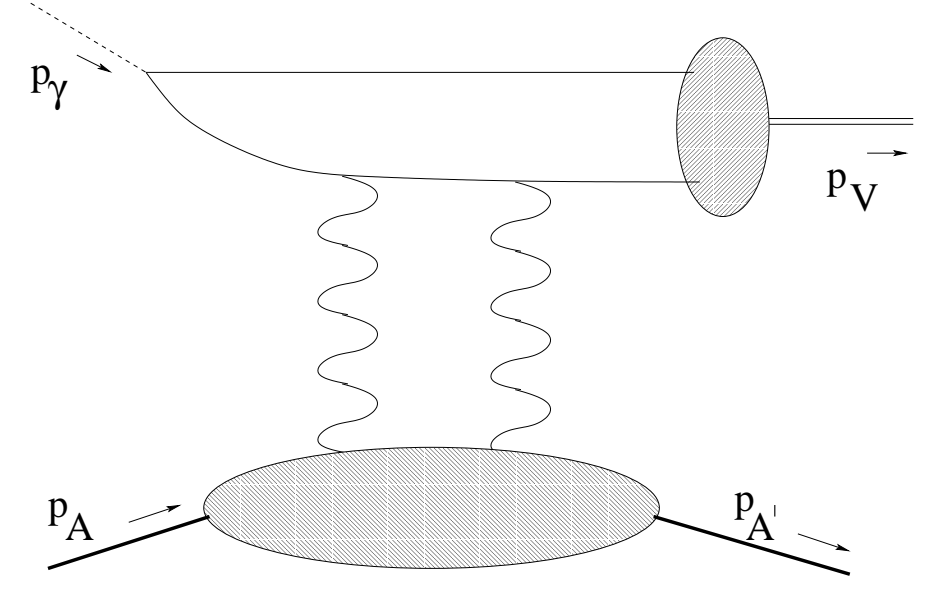

Figure 1: A typical graph for the elastic process $\gamma+A \rightarrow V+A^{\prime}$.

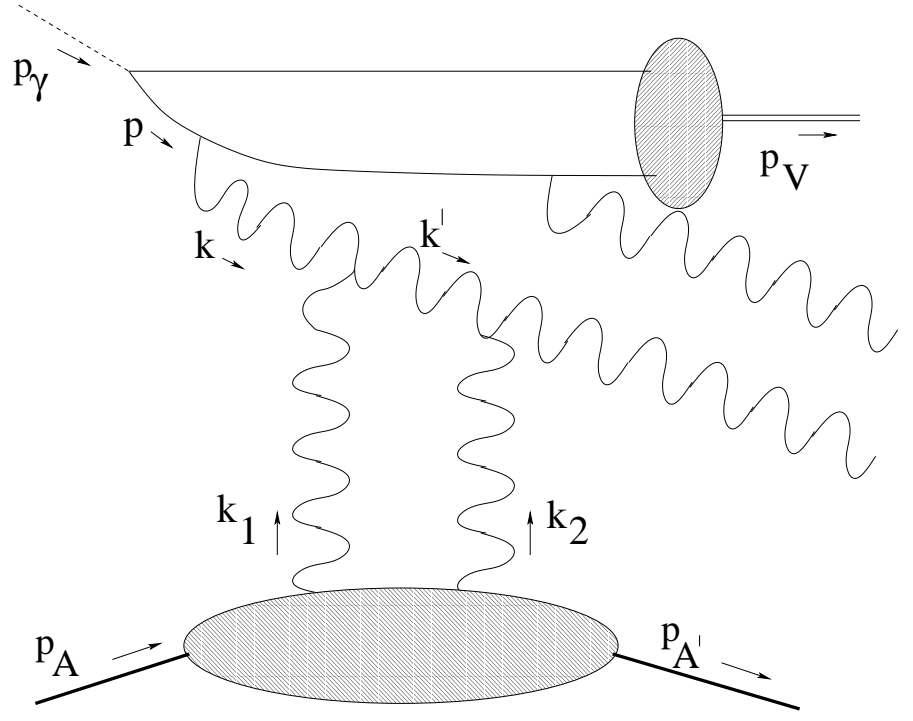

Figure 2: $Q \bar{Q} g$ contribution to diffractive quarkonium production.

In the long interval between the formation of the parton system and the interaction with the target's field, gluons can be radiated, possibly at small lightcone 
separations. These graphs contribute to evolution and radiative corrections. We will see later the result of including them.

Consider now the upper subgraph in Fig. 2, in which $k_{1}$ and $k_{2}$ couple to the $Q \bar{Q} g$ system. This subgraph is dominated by the region in which the transverse momentum $|\mathbf{p}|$ is of order $M$ (with $M$ the heavy meson mass) and much larger than the gluon transverse momenta $|\mathbf{k}|$ and $\left|\mathbf{k}^{\prime}\right|$. This region contributes a hard-scattering squared amplitude of order $\alpha \alpha_{s}^{2}$, that is, of the same order as the hard scattering in Fig. 1. By power counting in the coupling, the contributions in Figs. 1 and 2 are of the same size. Later on we will see that the contribution in Fig. 2 dominates the contribution in Fig. 1 by power counting in the hard-scattering scale.

The observation above indicates that in the dominant region the gluon $k$ goes near the mass shell. The process becomes sensitive to long distances, and involves the nonperturbative distribution of gluons in the hadron $A$, subject to the condition that the hadron is diffractively scattered. This distribution is depicted in Fig. 3. It is defined in terms of the gluon operator $\widetilde{G}$ introduced in Eq. (1.1) by the matrix elements

$$
\begin{aligned}
& \frac{d f_{g}}{d x_{\mathbb{P}} d t}\left(\beta, x_{\mathbb{P}}, t, \mu\right)=\frac{1}{(4 \pi)^{3} \beta x_{\mathbb{P}} p_{A}^{+}} \sum_{X} \int d y^{-} e^{i \beta x_{\mathbb{P}} p_{A}^{+} y^{-}} \\
& \times\left\langle A\left|\widetilde{G}_{a}(0)^{+j}\right| A^{\prime}, X\right\rangle\left\langle A^{\prime}, X\left|\widetilde{G}_{a}\left(0, y^{-}, \mathbf{0}\right)^{+j}\right| A\right\rangle
\end{aligned}
$$

with $\beta x_{\mathbb{P}}$ the fraction of the hadron's plus momentum carried by the gluon. These matrix elements have been studied in detail in $[15,16]$. The vertex at the top of Fig. 3 represents the gluon operator in Eq. (2.1), including a color-octet eikonal line defined by the path-ordered exponential in $\widetilde{G}[15,16]$. The eikonal line can be interpreted physically as a stand-in for the quark-antiquark pair that was produced by the electromagnetic current but is too compact to be resolved by the hadron's color field. Attachments of gluons to this line are incorporated as explained in [15].
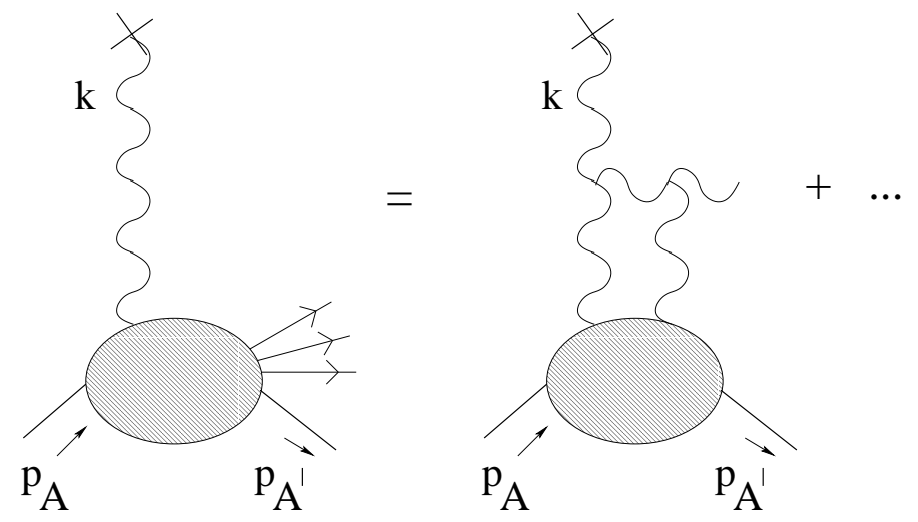

Figure 3: Diffractive matrix element for the gluon operator (Eq. (2.1)). The graph on the right hand side corresponds to the contribution depicted in Fig. 2. 


\section{The $t$-channel point of view}

The precise relation of the vector meson cross section with the matrix elements $(2.1)$ is given by a factorization formula. This can be obtained by applying to the graph of Fig. 2 standard methods $[17,18]$ for determining the leading-power asymptotics in the limit of a large hard-scattering scale.

The leading integration regions in momentum space involve (see Fig.4) i) a beam jet in the direction of hadron $A$, including the final hadron $A^{\prime}$; ii) a hard subgraph, in which all virtual lines are off shell by order $M$; iii) final states jets, including the vector meson, that are not in the direction of $A$; iv) a soft subgraph, consisting of soft gluons that link the beam jet with the hard subgraph and final state jets.

The method $[17,18]$ instructs us to a) use Ward identities to factor out soft gluon subgraphs from final-state and beam jets, and b) sum over real and virtual graphs to show that all soft gluon exchanges (dashed lines in Fig. 4) cancel in the cross section. This can be accomplished in a standard manner in the problem at hand, except for the treatment of soft gluons coupling to the beam jet in the plus direction: owing to the diffractive requirement on the final state, there are both initial-state and final-state couplings, giving poles on both sides of the integration contour in the complex plane of the gluon minus momentum $r^{-}$. This prevents us from deforming the $r^{-}$contour to the region where the soft approximation is valid.

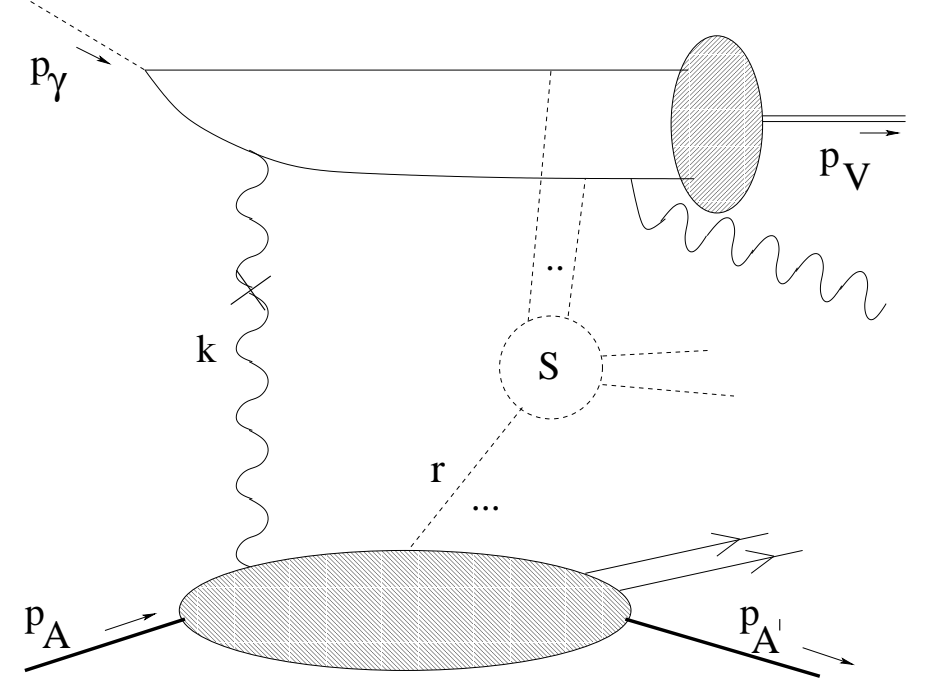

Figure 4: Factorization of the inelastic diffraction process. Soft gluon exchanges (dashed lines) cancel.

This obstruction, however, can be overcome in the same way as in diffractive deep inelastic scattering [19], by going to the complex plane of the plus momentum component $r^{+}$, and showing that in this plane the contour is not trapped by singularities in the beam jet subgraph. Then the cancellation of soft gluon exchanges goes through, and factorization follows. To keep the notation simpler we first consider 
the case $Q^{2}=0$, limiting ourselves to the direct-photon part of photoproduction. (We comment on the resolved-photon part below.) The factorization formula for the $\gamma+A \rightarrow V+X+A^{\prime}$ cross section reads

$$
\frac{d \sigma^{(\text {photo })}}{d x_{\mathbb{P}} d t}\left(M^{2}, W^{2}, x_{\mathbb{P}}, t\right)=\sum_{a=q, \bar{q}, g} \int_{\omega / x_{\mathbb{P}}}^{1} d \beta \frac{d f_{a}}{d x_{\mathbb{P}} d t}\left(\beta, x_{\mathbb{P}}, t, \mu^{2}\right) \hat{\sigma}_{a}\left(\frac{\omega}{\beta x_{\mathbb{P}}}, M^{2}, \mu^{2}\right)
$$

with $W^{2}=\left(p_{A}+p_{\gamma}\right)^{2}, \omega=M^{2} / W^{2}$. The coefficient $\hat{\sigma}_{a}$ describes the meson production subprocess represented by the upper subgraph in Fig. 4. In the nonrelativistic expansion for the bound state, $\hat{\sigma}_{a}$ factorizes into the product of a perturbative factor, describing the production of the quark-antiquark pair, and a nonperturbative factor, describing the binding of the quark and antiquark into a quarkonium. In this paper we consider only the lowest order in this expansion, corresponding to color-singlet quark-antiquark states [20]. We comment below on the possible role of color-octet states.

Both $\hat{\sigma}_{a}$ and $f_{a}$ in Eq. (3.1) depend on the factorization scale $\mu$. As noted earlier, the evolution in $\mu$ arises, via renormalization of the ultraviolet divergences, from gluons being radiated from the top subgraph in Fig. 2. We refer the reader to Refs. $[15,16,19,7]$ for detailed treatments of evolution in hard-diffractive processes.

To the leading order in $\alpha_{s}$, only the term with $a=g$ enters in the sum in Eq. (3.1). This is the term depicted in Figs. 2,4. Compare this with the case of the diffractive structure function $F_{2}$, where $a=g$ enters to the next-to-leading order, and of diffractive jet production, where both $a=g$ and $a=q$ enter to the leading order. (See, e.g., [21, 22] for early treatments of gluon radiation in these processes.) This makes the quarkonium cross section an especially important experimental probe of the physics of large gluon densities in diffraction.

The coefficient $\hat{\sigma}_{g}$ that relates the quarkonium cross section to the diffractive gluon density can be obtained from the perturbative calculations of [20] and reads

$$
\begin{aligned}
\hat{\sigma}_{g}\left(\omega, M^{2}, \mu^{2}\right)= & \frac{32}{3 \pi} \frac{\alpha e_{Q}^{2} \alpha_{s}^{2}\left(\mu^{2}\right)}{M^{2}} \frac{|\psi(0)|^{2}}{M^{3}} \omega \\
& \times\left\{\frac{1+\omega}{1-\omega}+\frac{1-\omega}{(1+\omega)^{2}}+2 \omega \ln \frac{1}{\omega}\left[\frac{1}{(1+\omega)^{3}}-\frac{1}{(1-\omega)^{2}}\right]\right\}
\end{aligned}
$$

Here $\psi(0)$ is the quarkonium wave function at the origin. Eq. (3.2) vanishes like $\omega$ for large energies $(\omega \rightarrow 0)$ and like $(1-\omega)$ near threshold $(\omega \rightarrow 1)$. In these boundary regions higher order corrections [23] to the coefficient become particularly important. Notice the behavior of the cross section (3.1) in the hard scale $M^{2}$. Apart from the dimensionless factor $|\psi(0)|^{2} / M^{3}$, coming from the nonrelativistic treatment of the bound state, $d \sigma / d t$ goes like $1 / M^{2}$. This is to be contrasted with the behavior $1 / M^{4}[4,6]$ of the elastic process (Fig. 1). That is, for sufficiently large $M$ the cross section is significantly larger than its elastic part. 
As noted above, this treatment does not include terms from color-octet quarkantiquark states. Color-octet terms can in general be large, because, although suppressed by powers of the heavy quark velocity, they are enhanced by a power of $1 / \alpha_{s}$ [24]. In this respect, they are more important in the inelastic-diffraction process than in the elastic case. Observe however that if the final state is diffractive the contribution of color-octet states does not necessarily follow the simple factorized form that is commonly used in applications of the nonrelativistic expansion. The soft gluons emitted in the transition of the color-octet quark pair to quarkonium may couple to the diffracted final state. These soft gluons are likely to reduce the probability for producing a quarkonium via a color-octet pair while leaving hadron $A$ intact. The net effect is that color-octet contributions should be suppressed in diffractive production compared to expectations valid in the fully inclusive case.

The resolved-photon part of photoproduction cannot be described by a factorization formula of the kind (3.1), because the possibility of color being exchanged with the photon beam jet spoils the cancellation of the soft gluon subgraphs. Since the direct and resolved parts cannot be separated in general beyond the leading logarithms, this limits the applicability of factorization in the $Q^{2}=0$ case.

Note however that, if we estimated the sign and order of magnitude of the soft contributions from diffractive hadron-hadron data (see, e.g., [25]), this would suggest that the relative importance of the resolved part compared to the direct part is smaller in diffractive photoproduction than in inclusive photoproduction, perhaps by a factor of several. This, combined with the use of kinematic cuts designed to suppress the resolved process, may allow one to make a meaningful test of the direct process.

\section{4. $Q^{2} \neq 0$ case}

The leptoproduction case $Q^{2} \neq 0$ is conceptually simpler than photoproduction, because it does not involve the processes with soft color exchange discussed at the end of the previous section. In the leptoproduction case a result of the kind (3.1) holds. More precisely, both the longitudinal and the transverse cross sections satisfy a factorization formula in terms of the diffractive matrix elements (2.1):

$$
\begin{aligned}
& \frac{d \sigma^{(\text {lepto })}}{d x_{\mathbb{P}} d t d Q^{2} d y}\left(M^{2}, S, Q^{2}, y, x_{\mathbb{P}}, t\right)=\frac{\alpha}{\pi} \frac{1}{y Q^{2}} \\
& \times\left[\left(1-y+\frac{y^{2}}{2}\right) \frac{d \sigma_{2}}{d x_{\mathbb{P}} d t}\left(M^{2}, Q^{2}, y S, x_{\mathbb{P}}, t\right)-\frac{y^{2}}{2} \frac{d \sigma_{L}}{d x_{\mathbb{P}} d t}\left(M^{2}, Q^{2}, y S, x_{\mathbb{P}}, t\right)\right] \\
& \frac{d \sigma_{i}}{d x_{\mathbb{P}} d t}=\sum_{a} \int d \beta \frac{d f_{a}}{d x_{\mathbb{P}} d t}\left(\beta, x_{\mathbb{P}}, t, \mu^{2}\right) \hat{\sigma}_{i, a}\left(\frac{\omega^{\prime}}{\beta x_{\mathbb{P}}}, M^{2}, Q^{2}, \mu^{2}\right) \quad(i=2, L)
\end{aligned}
$$


Here, in standard notation, $\sigma_{2}$ is the sum of the transverse and longitudinal cross

sections, $\sqrt{S}$ is the lepton-hadron center-of-mass energy, $y$ is the lepton energy loss in the target rest frame, and $\omega^{\prime}=\left(M^{2}+Q^{2}\right) /(y S)$.

Note that in the case of the elastic process (Fig. 1), in contrast, a factorization formula exists so far only for the longitudinal polarization [7].

The coefficients $\hat{\sigma}_{i, a}$ generalize Eq. (3.2) to $Q^{2} \neq 0$. Consider the power counting at large $Q$. (By comparison, recall $[5,6]$ that the elastic process of Fig. 1 scales like $1 / Q^{6}$ at large $Q$.) For $Q \gg M$, a factor of $1 / Q^{2}$ replaces the $1 / M^{2}$ of Eq. (3.2). Fig. 2 has a suppression factor $M^{2} / Q^{2}$ for producing a quarkonium of mass $M$ when the momentum flowing in the quark-antiquark subgraph is of order $Q$. However, note that for $Q \gg M$ Fig. 2 is no longer dominant, as contributions from the intrinsic heavy-quark distribution and from parton fragmentation into quarkonium scale like $1 / Q^{2}$. We therefore suggest considering the region of $Q$ of order $M$, or smaller than $M$. In this region, quark-antiquark-gluon states give a contribution to diffractive quarkonium production that is both leading in powers of the hard scale and leading in the running coupling.

Detailed studies are warranted to assess the numerical importance of this contribution at HERA and future $e A$ colliders.

\section{Discussion}

We have seen that the electroproduction of quarkonia by inelastic diffraction measures, through the factorization formulas of Secs.3 and 4, the diffractive gluon distribution (2.1). For $Q$ of order $M$, or smaller than $M$, this process provides a remarkable probe of quark-antiquark-gluon states in the photon lightcone wave function. Recall that, in contrast, the production of quarkonia by elastic diffraction couples predominantly to quark-antiquark states.

The differences between the dynamics of $Q \bar{Q}$ and $Q \bar{Q} g$ states scattering off the hadron's soft color field have been investigated extensively in the color dipole approximation (see, e.g., $[12,13,14]$, and references therein). Color-octet dipoles interact more strongly with the target. As a result, the probability for the parton system to get through the hadron without breaking it up dies off faster as the dipole size increases, and the hadron starts to look opaque earlier.

Based on the analysis of these effects, a physical picture for the diffractive parton distributions was suggested in [26]. One of the implications of the onset of color opacity is that, if the maximum parton separation $1 / \kappa$ for which the hadron is transparent is sufficiently small, a perturbative calculation [27] of the $\beta$ dependence of the diffractive distributions should apply. Such a calculation is incorporated in [26] and in different models $[28,29]$ for the diffractive distributions. The overall normalization of the distributions is inversely proportional to the square of the transparency length $1 / \kappa$ (in units of the hadron radius $r_{A}$ ), while their shape in $\beta$ is given by 
perturbatively computable functions $\varphi$ :

$$
f_{g} \propto\left(N_{c}^{2}-1\right) \kappa_{g}^{2} r_{A}^{2} \varphi_{g}(\beta), \quad f_{q} \propto N_{c} \kappa_{q}^{2} r_{A}^{2} \varphi_{q}(\beta) .
$$

In [26] predictions based on these distributions were compared with the HERA data [30] for the diffractive structure function $F_{2}$. The comparison indicates that transparency lengths are smaller for $Q \bar{Q} g$ states than for $Q \bar{Q}$ states. The $F_{2}$ data are consistent with $\kappa_{g} / \kappa_{q} \approx C_{A} / C_{F}$. Note that, together with the color factors explicitly shown in (5.1), this value gives back the result [27] for the ratio of the diffractive gluon and quark distributions in a small dipole,

$$
\frac{f_{g}}{f_{q}} \propto \frac{C_{A}^{2}\left(N_{c}^{2}-1\right)}{C_{F}^{2} N_{c}}=\frac{27}{2},
$$

thus supporting the picture based on the dominance of small parton separations. But a probe that, unlike $F_{2}$, couples directly to gluons will give a much better way of exploring this picture. The vector meson cross sections considered in this paper, Eqs. (3.1) and (4.2), will allow one to study precisely the relation between the large size of the diffractive gluon distribution in a proton and the dominance of small transparency lengths.

Note that this study may also be critical for deep inelastic scattering on nuclei, if information extracted from diffractive processes is to be used to compare approaches to nuclear shadowing [31].

It is worth commenting on the $x_{\boldsymbol{P}}$ dependence. In conventional fits to diffractive data, a simple factorizing form of the $x_{\boldsymbol{P}}$ and $\beta$ dependence is often assumed. From the QCD point of view, this is an ansatz, not a theory result. In particular, notice that the diffractive gluon and quark distributions at low $\mu$ need not have the same $x_{P}$ dependence. Therefore, even if a simple factorizing form $x_{\boldsymbol{P}}^{1-2 \alpha}$ is taken, it would be interesting to analyze the data using two different behaviors for the gluon and quark distributions, parameterized by $\alpha_{g}$ and $\alpha_{q}$. The heavy meson cross section discussed in this paper would provide a good way of determining $\alpha_{g}$.

\section{Acknowledgments}

I gratefully acknowledge the hospitality and support of the Institute for Nuclear Theory at the University of Washington while part of this work was being done. I thank J. Collins, L. Frankfurt, D. Soper and M. Strikman for useful discussions. This research is funded in part by the US Department of Energy grant DE-FG0290ER40577. 


\section{References}

[1] ZEUS Collaboration (J. Breitweg et al.), Eur. Phys. J. C6, 603 (1999).

[2] H1 Collaboration (C. Adloff et al.), Eur. Phys. J. C10, 373 (1999).

[3] H1 Collaboration (C. Adloff et al.), Phys. Lett. B 483, 23 (2000).

[4] M.G. Ryskin, Z. Phys. C 57, 89 (1993); M.G. Ryskin, R.G. Roberts, A.D. Martin and E.M. Levin, Z. Phys. C 76, 231 (1997).

[5] S.J. Brodsky, L. Frankfurt, J.F. Gunion, A.H. Mueller and M. Strikman, Phys. Rev. D 50, 3134 (1994).

[6] L. Frankfurt, W. Koepf and M. Strikman, Phys. Rev. D 57, 512 (1998).

[7] J.C. Collins, L. Frankfurt and M. Strikman, Phys. Rev. D 56, 2982 (1997).

[8] D. Müller, D. Robaschik, B. Geyer, F.-M. Dittes and J. Hořejši, Fortsch. Phys. 42, 101 (1994).

[9] X.D. Ji, Phys. Rev. D 55, 7114 (1997).

[10] A. Radyushkin, Phys. Lett. B 385, 333 (1996).

[11] J.D. Bjorken, AIP Conference Proceedings No. 6, Particles and Fields subseries No. 2 (New York 1972); hep-ph/9601363; J.D. Bjorken, J. Kogut and D.E. Soper, Phys. Rev. D 3, 1382 (1971).

[12] N.N. Nikolaev and B.G. Zakharov, Z. Phys. C 49, 607 (1991), ibid C 53, 331 (1992); A.H. Mueller, Nucl. Phys. B335, 115 (1990); L. Frankfurt and M. Strikman, Phys. Rep. 160, 235 (1988).

[13] A.H. Mueller, in Proceedings of the International Workshop on Deep Inelastic Scattering DIS98 (Brussels, April 1998), World Scientific, Singapore 1998, p.3.

[14] L. Frankfurt and M. Strikman, in Proceedings of the International Workshop on Deep Inelastic Scattering DIS99 (Zeuthen), Nucl. Phys. B Proc. Suppl. 79, 671 (1999).

[15] F. Hautmann, Z. Kunszt and D.E. Soper, Nucl. Phys. B563, 153 (1999).

[16] A. Berera and D.E. Soper, Phys. Rev. D 53, 6162 (1996).

[17] S.B. Libby and G. Sterman, Phys. Rev. D 18, 3252 (1978); ibid. 18, 4737 (1978).

[18] J.C. Collins and D.E. Soper, Ann. Rev. Nucl. Part. Sci. 37, 383 (1987).

[19] J.C. Collins, Phys. Rev. D 57, 3051 (1998), (E) ibid. D 61, 019902 (2000).

[20] E.L. Berger and D. Jones, Phys. Rev. D 23, 1521 (1981); R. Baier and R. Rückl, Z. Phys. C 19, 251 (1983). 
[21] N.N. Nikolaev and B.G. Zakharov, Z. Phys. C 64, 631 (1994).

[22] J. Bartels, H. Jung and M. Wüsthoff, Eur. Phys. J. C11, 111 (1999).

[23] M. Krämer, J. Zunft, J. Steegborn and P.M. Zerwas, Phys. Lett. B 348, 657 (1995); M. Krämer, Nucl. Phys. B459, 3 (1996).

[24] M. Cacciari and M. Krämer, Phys. Rev. Lett. 76, 4128 (1996); P. Ko, J. Lee and H.S. Song, Phys. Rev. D 54, 4312 (1996), (E) ibid. D 60, 119902 (1999); J. Amundson, S. Fleming and I. Maksymyk, Phys. Rev. D 56, 5844 (1997).

[25] M.G. Albrow, in Proceedings of the International Symposium on Multiparticle Dynamics, Tihany, Hungary, October 2000, hep-ph/0102092.

[26] F. Hautmann and D.E. Soper, Phys. Rev. D 63, 011501 (2000).

[27] F. Hautmann, Z. Kunszt and D.E. Soper, Phys. Rev. Lett. 81, 3333 (1998).

[28] W. Buchmüller, T. Gehrmann and A. Hebecker, Nucl. Phys. B537, 477 (1999).

[29] K. Golec-Biernat and M. Wüsthoff, Eur. Phys. J. C20, 313 (2001).

[30] ZEUS Collaboration (J. Breitweg et al.), Eur. Phys. J. C6, 43 (1999); H1 Collaboration (C. Adloff et al.), Z. Phys. C76, 613 (1997).

[31] L. Frankfurt, V. Guzey, M. McDermott and M. Strikman, hep-ph/0201230. 